\title{
DETECTION AND PARTIAL GENETIC CHARACTERISATION OF NOVEL AVI- AND SIADENOVIRUSES IN RACING AND FANCY PIGEONS (COLUMBA LIVIA DOMESTICA)
}

\author{
Mónika Z. BALLMANN and Balázs HARRACH* \\ Institute for Veterinary Medical Research, Centre for Agricultural Research, \\ Hungarian Academy of Sciences, P.O. Box 18, H-1581 Budapest, Hungary
}

(Received 15 August 2016; accepted 2 November 2016)

Up to now, only a single adenovirus (AdV) isolate seemingly specific for pigeons, hence named pigeon AdV-1 (PiAdV-1), has been characterised at DNA sequence level. In the present work, the prevalence and diversity of AdVs occurring in domestic pigeon were examined by a survey performed on randomly collected samples using a very efficient, consensus nested PCR targeting the viral DNA polymerase gene. The newly detected viruses were characterised by sequencing and phylogeny analysis. Amplification of additional genome fragments was attempted by the use of several other PCR methods aiming at the hexon gene. During a 4-year survey, samples from dead or live, healthy pigeons originating from 27 lofts were examined in Hungary. Almost $50 \%$ of the samples (48 out of 97) proved to be positive for AdV. Sequence analysis revealed the presence of four hitherto unknown pigeon AdV types. PiAdV-1 was also identified in one sample. Two novel viruses named PiAdV-2 and -3 were found to belong to the genus Aviadenovirus, and two other novel types (PiAdV-4 and -5) to the genus Siadenovirus. This is the first report on the occurrence of siadenoviruses in birds belonging to the order Columbiformes. Approximately two-thirds of the PiAdV-2 genome was sequenced and analysed. PCR

Key words: Pigeon, adenovirus, aviadenovirus, siadenovirus, phylogeny,

Family Adenoviridae is divided into five genera (Mastadenovirus, Aviadenovirus, Atadenovirus, Siadenovirus and Ichtadenovirus). The presence of adenoviruses (AdVs) has been described in representatives of a large number of vertebrate species including primates as well as fish, amphibians and reptiles (Harrach et al., 2011). Birds are known to be common hosts for AdVs and this fact was mirrored by the large number of the so-called fowl AdVs (FAdVs) described from chicken already several decades ago (Smyth and McNulty, 2008). Moreover, birds are among the few hosts that can be infected by rather divergent

*Corresponding author; E-mail: harrach.balazs@agrar.mta.hu; Phone: 0036 (1) 467-4081; Fax: 0036 (1) 467-4076 
AdVs classified into three different genera, namely Aviadenovirus, Siadenovirus and Atadenovirus.

Aviadenoviruses have been detected almost exclusively in birds and this genus is thought to have cospeciated with the class of Aves during evolution (Harrach and Kaján, 2011). Twelve FAdV serotypes (FAdV-1 to 8a, 8b to 11) are grouped into five species, named Fowl aviadenovirus $A$ to $E$ (informally abbreviated as $F A d V-A-E$ ) (Harrach and Kaján, 2011; Kaján et al., 2011). Additional aviadenovirus serotypes have been described from goose, duck and turkey (Kaján et al., 2012; Marek et al., 2014 $a$ and $b$ ). As to wild birds, aviadenoviruses have been found in representatives of the orders Falconiformes (Schrenzel et al., 2005), Psittaciformes (Raue et al., 2005a; Wellehan et al., 2005) and Charadriiformes (Bodewes et al., 2013). From Columbiformes, a single AdV named pigeon adenovirus 1 (PiAdV-1), has been characterised by DNA sequence (Hess et al., 1998; Marek et al., 2014b).

The presence of atadenoviruses and siadenoviruses in birds has been hypothesised to be the result of interclass host switches (Benkö and Harrach, 2003). Atadenoviruses presumably have coevolved with squamate reptiles (order Squamata) (Pénzes et al., 2014). The first members of the genus Siadenovirus were discovered in poultry showing different signs of serious disease (Smyth and McNulty, 2008), as well as in a frog (Davison et al., 2000). While the number of siadenoviruses detected in a wide range of birds is increasing (Zsivanovits et al., 2006; Kovács et al., 2010; Park et al., 2012; Lee et al., 2014), there are only two approved members of the genus Atadenovirus known to be capable of infecting birds. Pigeons have not been found to harbour AdVs from any of these two genera until now.

Adenoviral infection in pigeons was first reported from Belgium in 1984 (Coussement et al., 1984), but by now it has been observed all over the world. Evidences for the presence of $\mathrm{AdV}$ in pigeons were compiled by electron microscopy, histology and serology (de Herdt et al., 1995; Vereecken et al., 1998). De Herdt et al. (1995) described two different disease manifestations that had been suspected to be caused by specific pigeon AdVs. According to their classification, type I or classical adenovirosis affects predominantly fledglings during their first racing season. The clinical signs include anorexia, enteritis, vomiting and diarrhoea. Postmortem examination reveals inclusion body hepatitis. This disease often occurs in association with Escherichia coli infection. The so-called type II adenovirosis or necrotising hepatitis is not age dependent and appears sporadically. The clinical signs affect the respiratory tract and the most frequent pathological finding is hepatic necrosis. A third condition, in which the potential role of AdVs has been examined, is the so-called Young Pigeon Disease Syndrome (YPDS). This multifactorial disease complex affects the age group of 4-12 weeks. Atypical clinical signs include anorexia, depression, ruffled feathers, vomiting and a fluid-filled crop. Generally, less than $20 \%$ of the young birds are affected but morbidity and mortal- 
ity might sometimes reach $50 \%$. Raue et al. $(2005 \mathrm{~b})$ suggested that the primary aetiological agent of YPDS is circovirus by causing depletion of the lymphoid tissues and immunosuppression. Secondary bacterial infections were also found to contribute to the pathogenesis of YPDS, but the presence of AdVs could not be confirmed by PCR. It has to be added that the primers used for these examinations were not universal but rather specific for FAdVs and PiAdV-1 (Meulemans et al., 2001; Raue et al., 2002). In other surveys, AdVs have been detected in a few cases only (Freick et al., 2008; Stenzel et al., 2012).

Initial attempts to isolate AdVs from pigeons have been made by using primary chicken cells, consequently almost every known FAdV type has been obtained (McFerran et al., 1976; Vereecken et al., 1998). The first distinct pigeon AdV strain was reported by Hess et al. (1998). On chicken embryo liver cells, they isolated a virus that was found to be a serologically novel type. This was further supported by the sequence of the PCR-amplified hexon gene fragment. The virus was named as PiAdV-1 and its whole genome sequence has been published recently (Marek et al., 2014b).

Our aim was to examine the prevalence and diversity of AdVs present in Hungarian pigeon lofts. We performed a PCR screening by using a very sensitive nested method with highly degenerate primers that recognise the most conserved portion of the adenoviral DNA-dependent DNA polymerase (pol) gene (Wellehan et al., 2004). Phylogenetic analysis of the sequence of amplicons permits preliminary genus classification of the given AdV. Then additional PCRs appropriate for the detection of AdVs from the respective genera can be applied. We also intended to test the PCR methods recommended for the diagnosis of avian and pigeon AdVs previously. The viruses in the positive samples were further studied in order to determine the nucleotide (nt) sequence of longer gene fragments or preferably complete genes. Approximately two thirds of the genome of the most frequently detected, novel PiAdV type could be sequenced.

\section{Materials and methods}

\section{Origin of samples}

Almost 100 birds, including 78 racing and 19 fancy pigeons from 27 different lofts, were examined for the presence of AdVs from various regions of Hungary. Every loft experienced some clinical disease for shorter or longer periods. Samples from internal organs (lung, liver, kidney, intestine) or liver samples exclusively were collected and examined from 33 and 47 birds, respectively. The pigeons were either found dead or humanely euthanised because of severe disease. The clinical signs included anorexia, vomiting, diarrhoea, weight loss and ruffled feathers. Besides these 80 carcasses, 17 cloacal swabs from live, healthy specimens from three lofts were also tested. 
NOVEL ADENOVIRUSES DETECTED IN PIGEONS

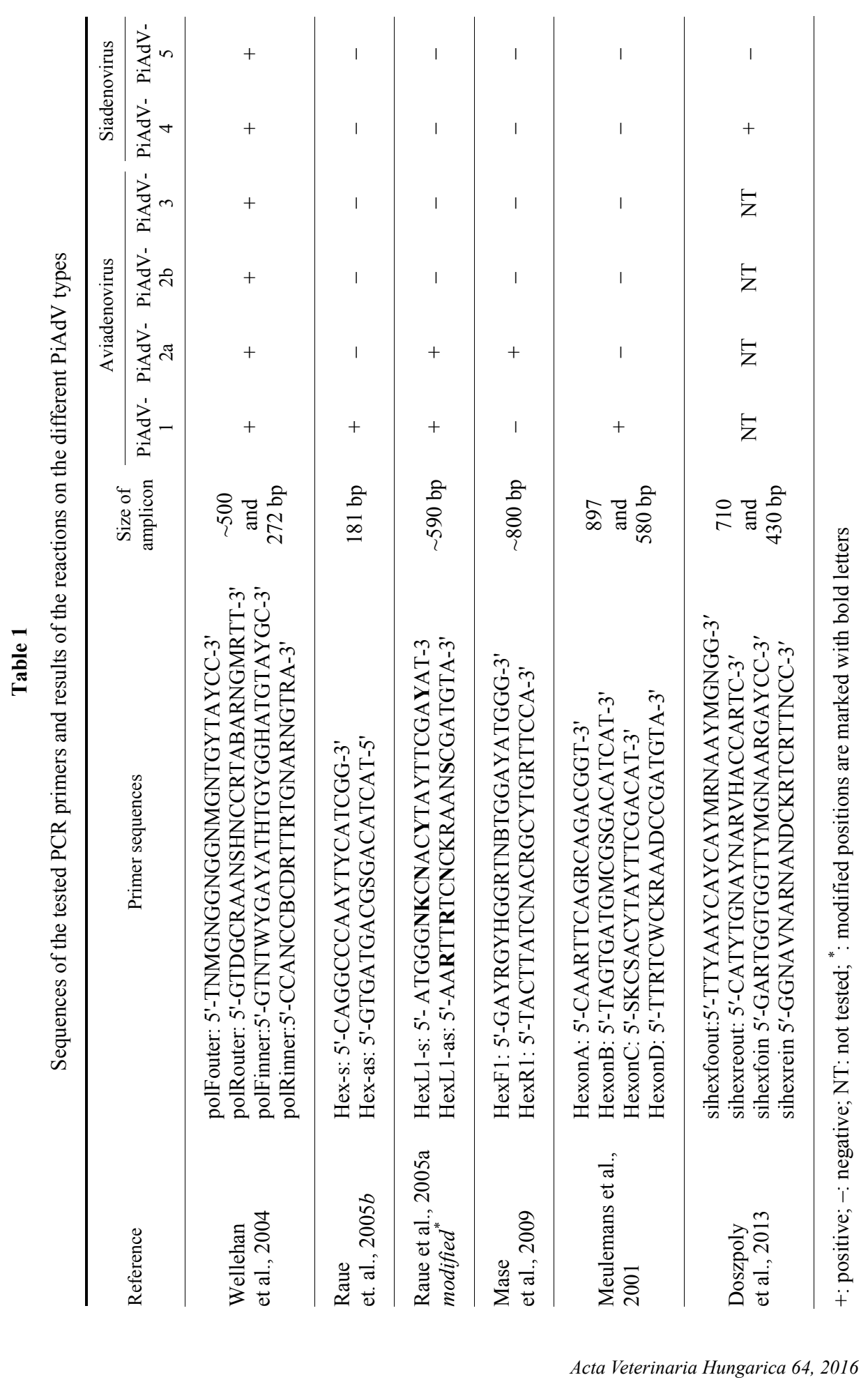


DNA extraction from the tissues was carried out as described recently (Doszpoly et al., 2014). For the excrements, the E.Z.N.A ${ }^{\circledR}$ Stool DNA Kit was used following the manufacturer's instructions (Omega BioTek).

\section{PCR and sequencing}

A consensus nested PCR targeting the pol gene was performed first (Wellehan et al., 2004). The positive samples were further tested by different PCRs depending on the virus type deduced from the partial pol sequences. Most of the additional (simple or nested) PCRs have been described for the amplification of fragments from the gene of the hexon, the major capsid protein (Meulemans et al., 2001; Raue et al., 2005 $a$ and $b$; Freick et al., 2008; Mase et al., 2009; Stenzel et al., 2012; Doszpoly et al., 2013). As shown in Table 1, we altered slightly the sequence of some primers in order to increase their sensitivity. Specific primers complementary to the already determined sequences of the newly detected AdVs were also designed for the use in primer walking sequencing strategy.

The PCRs were carried out in a TPersonal Thermocycler (Biometra, Göttingen, Germany) with REDTaq ${ }^{\circledR}$ ReadyMix ${ }^{\mathrm{TM}}$ PCR Reaction Mix (Sigma-Aldrich, St. Louis, MO, USA) according to the manufacturer's instructions. Different cycling programs, published along with the above-mentioned primers, were used. To check the results, $10-\mu 1$ aliquots of the reaction mixtures were electrophoresed in $1 \%$ agarose gels. The amplicons were purified with the NucleoSpin Gel and PCR Clean-up kit (Macherey-Nagel, Düren, Germany). Sequencing of the products was performed with the BigDye ${ }^{\mathbb{R}}$ Terminator v3.1 Cycle Sequencing Kit (Thermo Fisher Scientific) from both directions with the appropriate PCR primers.

\section{Primary sequence analysis and phylogenetic reconstructions}

For sequence editing and aligning, the Staden 7.1 Package and BioEdit 7.2.5 program were applied. For sequence identification, different Blast algorithms were used at the website of the NCBI. The deduced amino acid (aa) sequences were aligned by the ClustalW program with default parameters. Gaps and primer sequences were trimmed off in the GeneDoc 2.7. application. The Phylip program package was used to perform distance matrix analyses by applying JTT model, fitch (with global rearrangements) (http://mobyle.pasteur.fr, Pasteur Institute, Paris). Phylogeny reconstructions were visualised with Mega6.

\section{Genome sequencing and annotation}

Genome sequencing of the most frequently detected pigeon AdV type has been initiated by PCR amplification of some additional conserved gene fragments. The conserved target sequences were selected from alignments of different aviadenoviruses. Every DNA fragment was sequenced first directly to confirm the homogeneity of the respective PCR product. Longer genome fragments 
for sequencing were molecularly cloned after confirmations. For annotation and schematic presentation of the genome, the CLC program was used. The newly determined sequences were submitted to GenBank and assigned to accession numbers KX555529-KX555532, KX555534 and KX673408.

\section{Results}

Prevalence and diversity of adenoviruses in pigeon samples

Out of the 97 samples, 48 (49.5\%) were found positive for the presence of adenoviral DNA. The positivity rate showed slight fluctuation according to the type of samples (Table 2). From the 33 birds, from which multiple organs had been screened separately, $22(66.7 \%)$ proved to be positive, while from the 47 liver samples $17(36.2 \%)$ only. A surprisingly high proportion, $9(52.9 \%)$ out of the 17 cloacal samples of healthy birds were also positive.

Table 2

Results of the AdV screening PCR targeting the viral DNA polymerase gene

\begin{tabular}{lcccccccc}
\hline & $\begin{array}{c}\text { Pool } \\
\text { of four } \\
\text { organs }\end{array}$ & Liver & Lung & Kidney & Intestine & Liver & $\begin{array}{c}\text { Faeces/ } \\
\text { cloacal } \\
\text { swab }\end{array}$ & Total \\
\hline Tested & $\mathbf{3 3}$ & 33 & 33 & 33 & 33 & $\mathbf{4 7}$ & $\mathbf{1 7}$ & $\mathbf{9 7}$ \\
Positive & $\mathbf{2 2}$ & 10 & 17 & 14 & 14 & $\mathbf{1 7}$ & $\mathbf{9}$ & $\mathbf{4 8}$ \\
\hline PiAdV-1 & $\mathbf{1}$ & 1 & 1 & 0 & 1 & $\mathbf{0}$ & $\mathbf{0}$ & $\mathbf{1}$ \\
PiAdV-2a & $\mathbf{7}$ & 2 & 5 & 3 & 4 & $\mathbf{1 1}$ & $\mathbf{4}$ & $\mathbf{2 2}$ \\
PiAdV-2b & $\mathbf{2}$ & 0 & 1 & 0 & 2 & $\mathbf{6}$ & $\mathbf{0}$ & $\mathbf{8}$ \\
PiAdV-2a+2b & $\mathbf{3}$ & 2 & 3 & 2 & 2 & $\mathbf{0}$ & $\mathbf{0}$ & $\mathbf{3}$ \\
PiAdV-3 & $\mathbf{1}$ & 1 & 1 & 1 & 1 & $\mathbf{0}$ & $\mathbf{0}$ & $\mathbf{1}$ \\
\hline$-\cdots$ PiAdV-4 & $\mathbf{7}$ & 3 & 6 & 7 & 4 & $\mathbf{0}$ & $\mathbf{5}$ & $\mathbf{1 2}$ \\
PiAdV-5 & $\mathbf{1}$ & 1 & 0 & 1 & 0 & $\mathbf{0}$ & $\mathbf{0}$ & $\mathbf{1}$ \\
\hline Positivity (\%) & 66.7 & 30.3 & 51.5 & 42.4 & 42.4 & 36.2 & 52.9 & 49.5 \\
\hline
\end{tabular}

Every PCR product proved to be of adenoviral origin. Six different $\mathrm{pol}$ sequences were obtained. The phylogeny inference showed that four types of sequences were from aviadenoviruses and two from siadenoviruses. Among aviadenoviruses, the sequence of PiAdV-1 (Marek et al., 2014b) was identified in a single case. Another aviadenovirus, representing clearly a novel type was detected in 22 birds. Compared to this sequence, an almost identical pol sequence was found in 8 samples. Three additional samples seemed to contain both sequence variants. A fourth type of aviadenovirus pol sequence was obtained also just from a single sample. Siadenoviral pol sequences were identified in 13 cases; 
12 contained identical sequences and only one a second type of siadenovirus. The presence of DNA of FAdVs or any other known AdVs of poultry origin was not detected at all.

Table 2 presents the distribution of the positive samples according to the type of sample and AdV detected. For the most frequently found putative novel aviadenovirus, we propose the name pigeon adenovirus 2 (PiAdV-2). The two variants of the PiAdV-2 pol sequences differed from each other in 6 out of the $272 \mathrm{nt}$ only, yet had identical aa sequences. We named the more abundant variant PiAdV-2a, while the less frequent one PiAdV-2b. The divergence between the aa sequence of the corresponding pol fragments of PiAdV-1 and -2 was $23.3 \%$. The third type of aviadenoviral pol sequence, proposed to be named PiAdV-3, was detected in a single case only. It showed 26.7 and $27.8 \%$ aa sequence divergence compared to PiAdV-1 and -2 , respectively.

The frequently detected siadenoviral pol sequences showed no nt divergence on the 272-bp-long fragment. We propose the name PiAdV-4 for this putative virus. The pol sequence of the other putative siadenovirus, named PiAdV-5, differed from PiAdV-4 in $97 \mathrm{nt}$ on the 272-bp-long pol sequence (35.6\%), and was found in one specimen only.

As many of the 27 lofts were represented only by a single sample, we are not able to draw a firm conclusion about the distribution of the detected viruses on loft level (Table 3). However, loft 3 was represented by 8 samples out of which none showed AdV positivity, but we still think this sample number is not enough to conclude that this loft was free of AdV infections. We did not observe multiple infections within one specimen; however, we did see a mixed infection with the two variants of PiAdV-2 ( $a$ and $b$ ) in 3 specimens. Furthermore, we confirmed the presence of both PiAdV-2 and -4 within the same loft in 4 cases (lofts $1,4,10$ and 21).

\section{Phylogenetic place of pigeon adenoviruses}

The phylogenetic tree, based on the pol aa sequences, showed PiAdV-2 and -3 constituting a monophyletic group with PiAdV-1 within the genus Aviadenovirus, whereas PiAdV-4 and -5 belonging to genus Siadenovirus (Fig. 1). Interestingly, these latter PiAdVs are not monophyletic though both of them appeared clearly in the clade of avian siadenoviruses.

\section{Further characterisation of certain novel AdVs}

Amplification of the hexon gene fragment from the newly found PiAdVs was successful only with samples containing PiAdV-2a or -4 (Table 1). Since different primer sets gave positive reaction, comparison of the hexon gene fragments was not possible. Nonetheless, the partial hexon sequences further supported the distinctness and genus affiliation of the respective viruses. 
Table 3

Distribution of AdV-positive samples among pigeon lofts based on the results of PCR targeting the viral DNA polymerase gene

\begin{tabular}{|c|c|c|c|c|c|c|c|}
\hline Loft & $\begin{array}{c}\text { Total } \\
\text { samples }\end{array}$ & $\begin{array}{c}\text { AdV } \\
\text { positive }\end{array}$ & PiAdV-1 & PiAdV-2 & PiAdV-3 & PiAdV-4 & PiAdV-5 \\
\hline 1 & 45 & 22 & & 21 & & 1 & \\
\hline 2 & 8 & 4 & & & & 4 & \\
\hline 3 & 8 & 0 & & & & & \\
\hline 4 & 4 & 3 & & 1 & & 2 & \\
\hline 5 & 1 & 0 & & & & & \\
\hline 6 & 1 & 0 & & & & & \\
\hline 7 & 1 & 1 & & 1 & & & \\
\hline 8 & 1 & 0 & & & & & \\
\hline 9 & 1 & 1 & & 1 & & & \\
\hline 10 & 3 & 2 & & 1 & & 1 & \\
\hline 11 & 1 & 0 & & & & & \\
\hline 12 & 1 & 1 & & & & 1 & \\
\hline 13 & 1 & 0 & & & & & \\
\hline 14 & 1 & 0 & & & & & \\
\hline 15 & 1 & 1 & & & 1 & & \\
\hline 16 & 1 & 1 & & 1 & & & \\
\hline 17 & 4 & 1 & & 1 & & & \\
\hline 18 & 1 & 1 & & 1 & & & \\
\hline 19 & 1 & 0 & & & & & \\
\hline 20 & 1 & 1 & & 1 & & & \\
\hline 21 & 3 & 3 & & 1 & & 2 & \\
\hline 22 & 2 & 1 & & & & 1 & \\
\hline 23 & 2 & 2 & & 2 & & & \\
\hline 24 & 1 & 0 & & & & & \\
\hline 25 & 1 & 1 & & & & & 1 \\
\hline 26 & 1 & 1 & & 1 & & & \\
\hline 27 & 1 & 1 & 1 & & & & \\
\hline Total & 97 & 48 & 1 & 33 & 1 & 12 & 1 \\
\hline
\end{tabular}

Partial genome sequence of pigeon adenovirus $2 a$

Approximately two thirds of the genome of the most frequently demonstrated novel virus, PiAdV-2a was sequenced. During annotation of the 31,314-bplong contig, 26 complete and 3 partial genes were identified. Among the familycommon genes, in the central part of the genome, a frequent trait of aviadenoviruses, i.e. the presence of two fibre genes of different sizes was identified. The schematic partial genome map is presented in Fig. 2. The genome organisation largely corresponded to that of PiAdV-1 (Marek et al., 2014b). Interestingly, however, every gene of PiAdV-2a seemed to encode a smaller protein than its counterpart in PiAdV-1. Among the few exceptions were ORF13 and the pVIII gene that 
had identical size in the two viruses, respectively. Furthermore, the two fibre genes of PiAdV-2a were significantly shorter than those in PiAdV-1. The shorter fibre1 of PiAdV-2a consists of 141 aa compared to 246 aa in PiAdV-1. This is a more than $40 \%$ difference. Fibre 2 of PiAdV-2a was found to contain 552 aa as opposed to 749 in PiAdV-1. The three major structural domains, including the tail, shaft and knob regions, could be recognised in the deduced aa sequence of fibre 2 only, while in fibre1 only the tail and the shaft domain can be found.

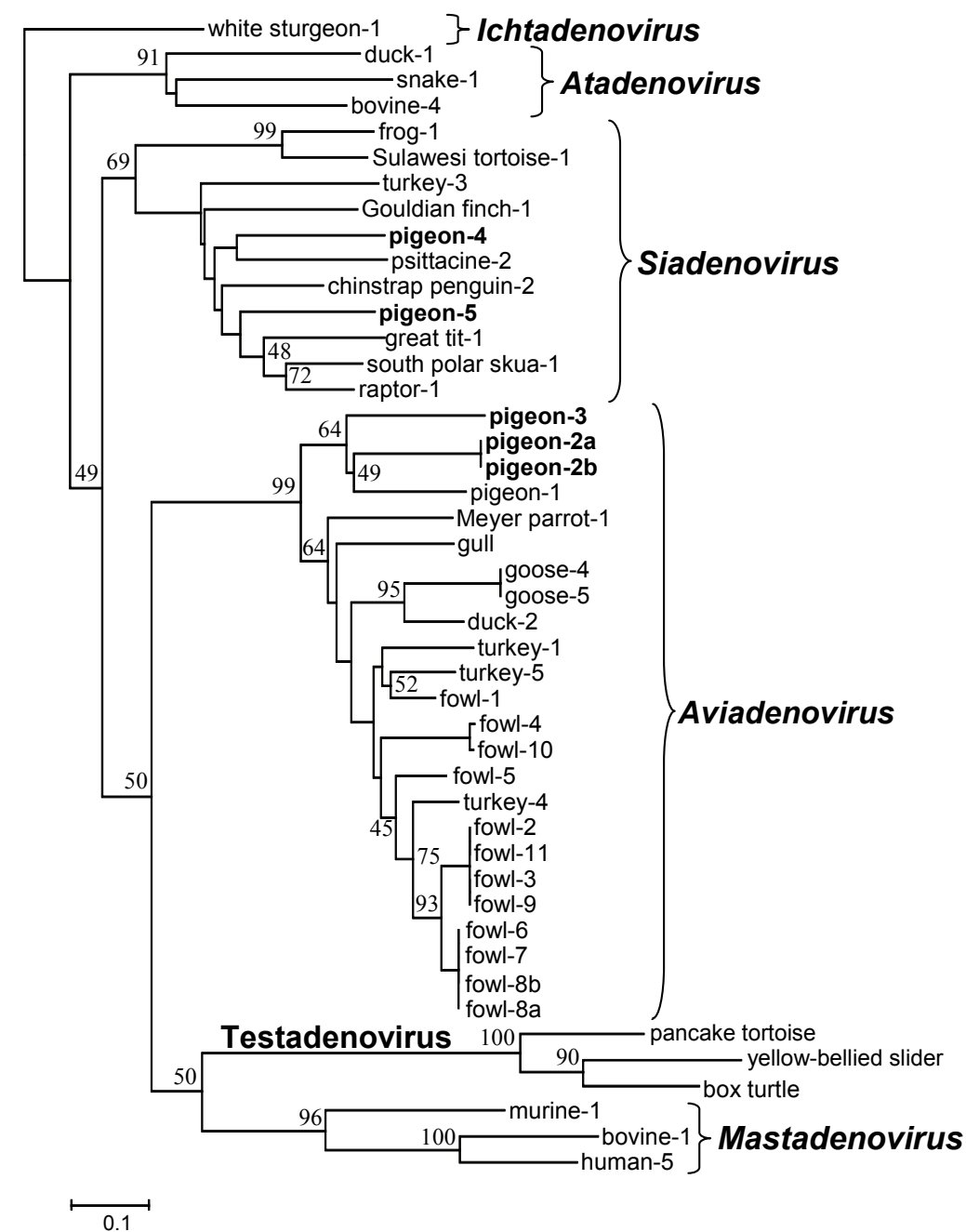

Fig. 1. Phylogenetic tree based on distance matrix analysis of the deduced amino acid sequences of the 272-bp-long DNA-dependent DNA polymerase gene fragments. Unrooted calculation.

For visualisation, the white sturgeon adenovirus 1 was selected as outgroup. Bootstrap values are based on 100 samplings. The viruses are marked by the host name and type number omitting the abbreviation AdV. The bar shows 0.1 nucleotide changes per site 
Splicing donor and acceptor sites were predicted in the genes of the terminal protein precursor (pTP), 33K and DNA-binding protein (DBP), as well as in ORF12, 13, 14 and 14A. Homologues of these ORFs are present in most aviadenovirus genomes studied to date. ORF1A and ORF20, flanking the studied genome portion, were sequenced incompletely. The short ORF corresponding to the so-called U exon was identified between the genes of pVIII and fibre1 (Fig. 2). The other exons belonging to the gene of the hypothetic ' $U$-exon protein' have not been identified in aviadenoviruses yet (Podgorski et al., 2016). No novel ORFs, specific for PiAdV-2a, were found in the examined genome fragment.

\section{Discussion}

Here, we describe the results of the first survey intended to assess the prevalence and diversity of AdVs in domestic pigeons in Hungary. The overall prevalence, found by PCR screening of almost 100 samples, was extremely high $(49.5 \%)$ especially if compared to the results of similar studies reported earlier from Germany and Poland, where only sporadic occurrence of PiAdVs was revealed (Raue et al., 2005b; Freick et al., 2008; Stenzel et al., 2012). The main reason behind the significant discrepancy is most likely the diverse performance of the PCR systems applied in the different screenings. We used a nested PCR with consensus primers that recognise the pol gene (Wellehan et al., 2004). The superiority of this PCR in detecting AdVs belonging to different genera has been documented in numerous cases in the past decade. It was originally designed for the recognition of novel atadenovirues in lizards, but its sensitivity and robustness allowed the discovery of many new aviadenoviruses (Wellehan et al., 2005; Bodewes et al., 2013), siadenoviruses (Katoh et al., 2009; Wellehan et al., 2009; Kovács et al., 2010; Park et al., 2012; Ballmann and Vidovszky, 2013; Joseph et al., 2014; Lee et al., 2014), atadenoviruses (Szirovicza et al., 2016) and mastadenoviruses (Vidovszky et al., 2015) in a wide range of hosts. Even the members of a hitherto unknown genus (with the proposed name Testadenovirus) could be detected (Doszpoly et al., 2013).

We revealed the existence of four novel AdV types in pigeons. The two aviadenoviruses, PiAdV-2 and -3 share close common ancestry with PiAdV-1. The partial genome analysis of PiAdV-2a revealed high similarity to PiAdV-1. These corroborate the genuine pigeon origin of all the three viruses. Nonetheless, the phylogenetic distances separating them merit the establishment of two novel species. PiAdV-1 is classified into the species Pigeon aviadenovirus A (Marek et al., 2014b). We propose that PiAdV-2 and -3 be members of Pigeon aviadenovirus $B$ and $C$, respectively. We also demonstrated the occurrence of PiAdV-1 in Hungary. 

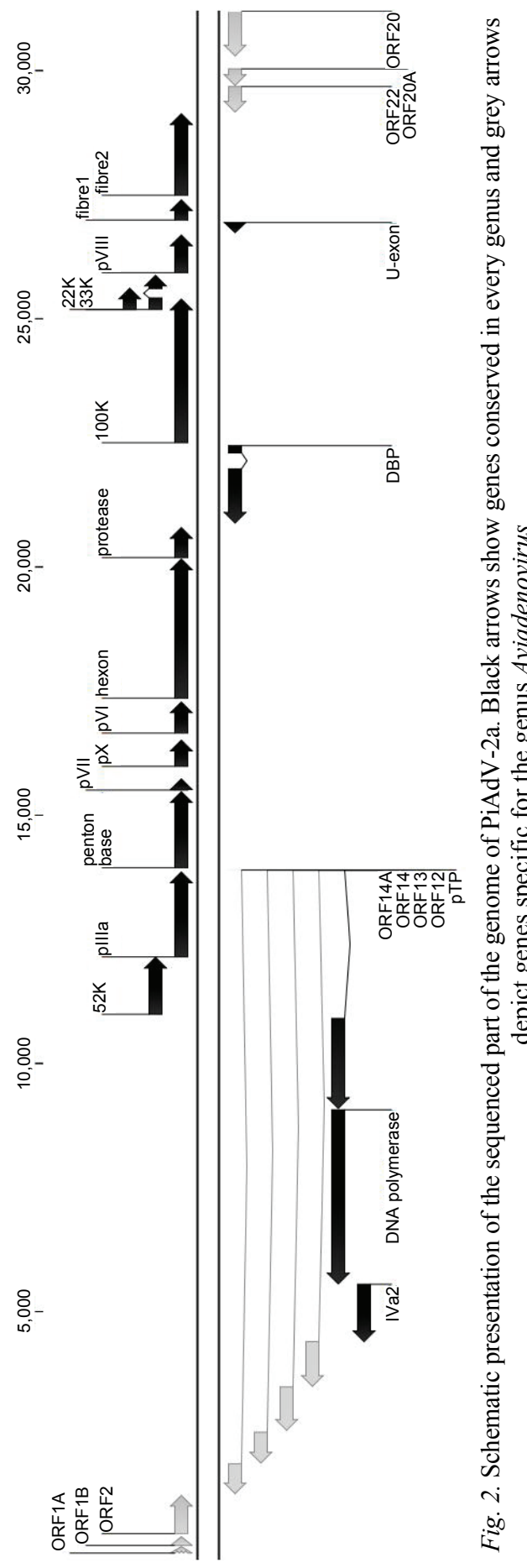
The evolutionary origin of siadenoviruses is yet unclear (Kovács and Benkö, 2011). Their presence in different birds is supposed to be the result of multiple host switch events. This theory is supported by the elevated pathogenicity and unusually wide host range of siadenoviruses (Benkő and Harrach, 2003; Wellehan et al., 2009; Ballmann and Vidovszky, 2013). The pathogenicity of the novel pigeon siadenoviruses described in this study has not been investigated yet. The large phylogenetic distance between PiAdV-4 and -5 implies that one or perhaps both of these viruses might have spread from some other host to pigeon.

In our experiments, the performance of the hexon-based PCRs was found to be poor. It seems that the level of variability of this gene does not support the design of primer sets universally applicable throughout the family Adenoviridae. The hexon can be a better target for genus- or type-specific PCRs. Even the nested primer set, originally designed on the basis of the most conserved aa stretches of the known siadenovirus hexons (Kovács, unpublished results) failed to recognise PiAdV-5. This was surprising, as this PCR had been successfully used for the demonstration of AdVs belonging to a different genus (Doszpoly et al., 2013).

The relationship between the newly found PiAdVs and YPDS or other disease conditions remains to be elucidated. In several recent papers, the crucial role of pigeon circovirus (PiCV) in YPDS was emphasised (Raue et al., 2005b; Stenzel et al., 2012). The prevalence of PiCV in healthy racing pigeons in Hungary was reported to be as high as $62 \%$ (Cságola et al., 2012). Our material originated predominantly from diseased and dead birds; therefore, it would be of special interest to find out the frequency of PiCV in these samples. We plan to perform such a PCR survey in the near future.

It is worth mentioning that we found $\mathrm{AdV}$-shedding birds among the healthy individuals in a considerable proportion (52.9\%). AdVs usually establish persistent infection, but virus shedding is not continuous, and infected birds may easily produce negative PCR results. Different stress factors, such as crowding, transportation, racing and most likely $\mathrm{PiCV}$ infection may trigger reactivation of AdVs, thus facilitating their PCR detection. Most of our samples were derived from racing pigeons. These birds are kept for hobby purposes and they participate in competitions on a regular basis. This means that they are regularly transported in crowded trucks or vans collecting pigeons from different lofts, and this stressful journey is followed by a challenging trip to the home loft on their own wings. Besides stress this is a regular way to exchange viral or bacterial agents between lofts.

Little is known about the tissue tropism of PiAdVs. As it can be deduced from the data in Table 2, the individual PiAdVs might replicate with different efficiency in the organs. Although the number of positive cases for each PiAdV did not allow statistical analysis yet, it is clear that sampling has a major impact on the outcome of diagnosis. Hepatitis or hepatic necrosis is commonly associated 
with AdVs in poultry, therefore the liver is often the only organ collected for PCR. It seemed that lung and kidney samples might be more appropriate especially in case of siadenovirus involvement.

In conclusion, our results show that at least five distinct PiAdVs circulate in the Hungarian domestic pigeon population. The complex aetiology of YPDS might deserve a closer look by using the most universal and robust pol PCR when searching for PiAdVs as possible secondary pathogens.

\section{Acknowledgements}

Financial support was provided by the Hungarian Scientific Research Fund (OTKA grant NN107632). The PhD fellowship to M. Z. B. by the Doctoral School of the Faculty of Veterinary Science, Szent István University is thankfully acknowledged. The authors thank Dr. Krisztián Berta and Dr. Ákos Thuma for providing pigeon samples, and Prof. Mária Benkő for her help in the compilation of the manuscript.

\section{References}

Ballmann, M. Z. and Vidovszky, M. Z. (2013): Detection of broad host range psittacine adenovirus (PsAdV-2) in different parrot species [in Hungarian, with English abstract]. Magy. Allatorvosok 135, 78-84.

Benkő, M. and Harrach, B. (2003): Molecular evolution of adenoviruses. Curr. Top. Microbiol. Immunol. 272, 3-35.

Bodewes, R., van de Bildt, M. W., Schapendonk, C. M., van Leeuwen, M., van Boheemen, S., de Jong, A. A., Osterhaus, A. D., Smits, S. L. and Kuiken, T. (2013): Identification and characterization of a novel adenovirus in the cloacal bursa of gulls. Virology 440, 84-88.

Coussement, W., Ducatelle, R., Lemahieu, P., Froyman, R., Devriese, L. A. and Hoorens, J. (1984): Pathologie van adenovirus infecties bij duiven [in Dutch]. Vleems Diergeneeskundig Tijdschrift 53, 227-283.

Cságola, A., Lőrincz, M., Tombácz, K., Wladár, Zs., Kovács, E. and Tuboly, T. (2012): Genetic diversity of pigeon circovirus in Hungary. Virus Genes 44, 75-79.

Davison, A. J., Wright, K. M. and Harrach, B. (2000): DNA sequence of frog adenovirus. J. Gen. Virol. 81, 2431-2439.

de Herdt, P., Ducatelle, R., Lepoudre, C., Charlier, G. and Nauwynck, H. (1995): An epidemic of fatal hepatic necrosis of viral origin in racing pigeons (Columba livia). Avian Pathol. 24, $475-483$.

Doszpoly, A., Tarján, Z. L., Glávits, R., Müller, T. and Benkő, M. (2014): Full genome sequence of a novel circo-like virus detected in an adult European eel Anguilla anguilla showing signs of cauliflower disease. Dis. Aquat. Organ. 109, 107-115.

Doszpoly, A., Wellehan, J. F. X. Jr., Childress, A. L., Tarján, Z. L., Kovács, E. R., Harrach, B. and Benkö, M. (2013): Partial characterization of a new adenovirus lineage discovered in testudinoid turtles. Infect. Genet. Evol. 17, 106-112.

Freick, M., Müller, H. and Raue, R. J. (2008): Rapid detection of pigeon herpesvirus, fowl adenovirus and pigeon circovirus in young racing pigeons by multiplex PCR. J. Virol. Methods 148, 226-231. 
Harrach, B., Benkő, M., Both, G. W., Brown, M., Davison, A. J., Echavarría, M., Hess, M., Jones, M. S., Kajon, A., Lehmkuhl, H. D., Mautner, V., Mittal, S. K. and Wadell, G. (2011): Family Adenoviridae. In: King, A. M. Q., Adams, M. J., Carstens, E. B. and Lefkowitz, E. J. (eds) Virus Taxonomy: IXth Report of the International Committee on Taxonomy of Viruses. Elsevier, San Diego. pp. 125-141.

Harrach, B. and Kaján, G. L. (2011): Aviadenovirus. Adenoviridae. In: Tidona, C. A. and Darai, G. (eds) The Springer Index of Viruses. Springer-Verlag, New York. pp. 13-28.

Hess, M., Prusas, C. and Monreal, G. (1998): Growth analysis of adenoviruses isolated from pigeons in chicken cells and serological characterization of the isolates. Avian Pathol. 27, 196-199.

Joseph, H. M., Ballmann, M. Z., Garner, M. M., Hanley, C. S., Berlinski, R., Erdélyi, K., Childress, A. L., Fish, S. S., Harrach, B. and Wellehan, J. F. X. Jr. (2014): A novel siadenovirus detected in the kidneys and liver of Gouldian finches (Erythura gouldiae). Vet. Microbiol. 172, 35-43.

Kaján, G. L., Davison, A. J., Palya, V., Harrach, B. and Benkő, M. (2012): Genome sequence of a waterfowl aviadenovirus, goose adenovirus 4. J. Gen. Virol. 93, 2457-2465.

Kaján, G. L., Sameti, S. and Benkő, M. (2011): Partial sequence of the DNA-dependent DNA polymerase gene of fowl adenoviruses: a reference panel for a general diagnostic PCR in poultry. Acta Vet. Hung. 59, 279-285.

Katoh, H., Ohya, K., Kubo, M., Murata, K., Yanai, T. and Fukushi, H. (2009): A novel budgerigaradenovirus belonging to group II avian adenovirus of Siadenovirus. Virus Res. 144, 294-297.

Kovács, E. R. and Benkő, M. (2011): Complete sequence of raptor adenovirus 1 confirms the characteristic genome organization of siadenoviruses. Infect. Genet. Evol. 11, 1058-1065.

Kovács, E. R., Jánoska, M., Dán, Á., Harrach, B. and Benkő, M. (2010): Recognition and partial genome characterization by non-specific DNA amplification and PCR of a new siadenovirus species in a sample originating from Parus major, a great tit. J. Virol. Methods 163, $262-268$.

Lee, S. Y., Kim, J. H., Park, Y. M., Shin, O. S., Kim, H., Choi, H. G. and Song, J. W. (2014): A novel adenovirus in Chinstrap penguins (Pygoscelis antarctica) in Antarctica. Viruses 6, 2052-2061.

Marek, A., Ballmann, M. Z., Kosiol, C., Harrach, B., Schlötterer, C. and Hess, M. (2014a): Wholegenome sequences of two turkey adenovirus types reveal the existence of two unknown lineages that merit the establishment of novel species within the genus Aviadenovirus. J. Gen. Virol. 95, 156-170.

Marek, A., Kaján, G. L., Kosiol, C., Harrach, B., Schlötterer, C. and Hess, M. (2014b): Complete genome sequences of pigeon adenovirus 1 and duck adenovirus 2 extend the number of species within the genus Aviadenovirus. Virology 462-463, 107-114.

Mase, M., Mitake, H., Inoue, T. and Imada, T. (2009): Identification of group I-III avian adenovirus by PCR coupled with direct sequencing of the hexon gene. Avian Pathol. 71, 1239-1242.

McFerran, J. B., Connor, T. J. and McCracken, R. M. (1976): Isolation of adenoviruses and reoviruses from avian species other than domestic fowl. Avian Dis. 20, 519-524.

Meulemans, G., Boschmans, M., van den Berg, T. P. and Decaesstecker, M. (2001): Polymerase chain reaction combined with restriction enzyme analysis for detection and differentiation of fowl adenoviruses. Avian Pathol. 30, 655-660.

Park, Y. M., Kim, J. H., Gu, S. H., Lee, S. Y., Lee, M. G., Kang, Y. K., Kang, S. H., Kim, H. J. and Song, J. W. (2012): Full genome analysis of a novel adenovirus from the South Polar skua (Catharacta maccormicki) in Antarctica. Virology 422, 144-150.

Pénzes, J. J., Menéndez-Conejero, R., Condezo, G. N., Ball, I., Papp, T., Doszpoly, A., Paradela, A., Pérez-Berná, A. J., López-Sanz, M., Nguyen, T. H., van Raaij, M. J., Marschang, R. E., Harrach, B., Benkő, M. and San Martín, C. (2014): Molecular characterization of a lizard adenovirus reveals the first atadenovirus with two fibre genes and the first adenovirus with either one short or three long fibres per penton. J. Virol. 88, 11304-11314. 
Podgorski, I. I., Pantó, L., Papp, T., Harrach, B. and Benkö, M. (2016): Genome analysis of four Old World monkey adenoviruses supports the proposed species classification of primate adenoviruses and reveals signs of possible homologous recombination. J. Gen. Virol. 97, $1604-1644$.

Raue, R., Gerlach, H. and Müller, H. (2005a): Phylogenetic analysis of the hexon loop 1 region of an adenovirus from psittacine birds supports the existence of a new psittacine adenovirus (PsAdV). Arch. Virol. 150, 1933-1943.

Raue, R., Hafez, H. M. and Hess, M. (2002): A fiber-gene based polymerase chain reaction for specific detection of pigeon adenovirus. Avian Pathol. 31, 95-99.

Raue, R., Schmidt, V., Freick, M., Reinhardt, B., Johne, R., Kamphausen, L., Kaleta, F. E., Müller, H. and Krautwald-Junghanns, M. E. (2005b): A disease complex associated with pigeon circovirus infection disease syndrome. Avian Pathol. 34, 418-425.

Schrenzel, M., Oaks, J. L., Rotstein, D., Maalouf, G., Snook, E., Sandfort, C. and Rideout, B. (2005): Characterization of a new species of adenovirus in falcons. J. Clin. Microbiol. 43, 3402-3413.

Smyth, J. A. and McNulty, M. S. (2008): Adenoviridae. In: Pattison, M., McMullin, P. F., Bradbury, J. M., Alexander, D. J. (eds) Poultry Diseases. Elsevier, Edinburgh. pp. 367-381.

Stenzel, T. A., Pestka, D., Tykalowsky, B., Smialek, M. and Koncicki, A. (2012): Epidemiological investigation of selected pigeon viral infections in Poland. Vet. Rec. 171, 562.

Szirovicza, L., López, P., Kopena, R., Benkő, M., Martín, J. and Pénzes, J. J. (2016): Random sampling of squamate reptiles in Spanish natural reserves reveals the presence of novel adenoviruses in lacertids (family Lacertidae) and worm lizards (Amphisbaenia). PLoS One 11, e0159016.

Vereecken, M., de Herdt, P. and Ducatelle, R. (1998): Adenovirus infection in pigeons: a review. Avian Pathol. 27, 333-338.

Vidovszky, M. Z., Kohl, C., Boldogh, S., Görföl, T., Wibbelt, G., Kurth, A. and Harrach, B. (2015): Random sampling of the European bat fauna reveals the existence of numerous hitherto unknown adenoviruses. Acta Vet. Hung. 63, 508-525.

Wellehan, J. F. X. Jr., Greenacre, C. B., Fleming, G. J., Stetter, M. D., Childress, A. L. and Terrell, S. P. (2009): Siadenovirus infection in two psittacine bird species. Avian Pathol. 38, 413-417.

Wellehan, J. F. X. Jr., Johnson, A. J., Harrach, B., Benkő, M., Pessier, A. P., Johnson, C. M., Garner, M. M., Childress, A. and Jacobson, E. R. (2004): Detection and analysis of six lizard adenoviruses by consensus primer PCR provides further evidence of reptilian origin for the atadenoviruses. J. Virol. 78, 13366-13369.

Wellehan, J. F. X., Johnson, A. J., Latimer, K. S., Bischoff, K., Lafortune, M. and Jacobson, E. R. (2005): Initial characterization of an adenovirus associated with fatal hepatic and lymphoid necrosis in a Meyer's parrot (Poicephalus meyeri). J. Avian Med. Surg. 19, 191-197.

Zsivanovits, P., Monks, D. J., Forbes, N. A., Ursu, K., Raue, R. and Benkő, M. (2006): Presumptive identification of a novel adenovirus in a Harris hawk (Parabuteo unicinctus), a Bengal eagle owl (Bubo bengalensis), and a Verreaux's eagle owl (Bubo lacteus). J. Avian Med. Surg. 20, 105-112. 